\title{
Análise centesimal e microbiológica das carcaças de bovinos e bubalinos de dois abatedouros de Macapá-Amapá
}

Centesimal and microbiological analysis of bovine and buffalo carcasses from two abattoirs in Macapá-Amapá

Análisis centesimal y microbiológico de canales de bovinos y bufalinos de dos mataderos de Macapá-Amapá

Cássia Meina Marques dos Reis Pereira ORCID: https://orcid.org/0000-0001-7621-1611 Faculdade Estácio SEAMA, Brasil E-mail: cassiameina@gmail.com

Paula Inara Tavares da Silva ORCID: https://orcid.org/0000-0002-9612-7924 Faculdade Estácio SEAMA, Brasil E-mail: paulatvrs1@gmail.com Jaqueline Freitas Souza

ORCID: https://orcid.org/0000-0002-3046-3212 Universidade Estadual do Maranhão, Brasil E-mail: jackllinefn@hotmail.com

Odonei Moia de Almeida

ORCID: https://orcid.org/0000-0003-0519-3190 Agência de Defesa e Inspeção Agropecuária do Estado do Amapá, Brasil E-mail: odonei@hotmail.com

Anne do Socorro Santos da Silva ORCID: https://orcid.org/0000-0001-5524-8095 Instituto de Pesquisas Científicas e Tecnológicas do Estado do Amapá, Brasil E-mail: anne.socorro@gmail.com

Jorge Belém Oliveira Júnior

ORCID: https://orcid.org/0000-0002-8130-1598

Universidade Federal de Pernambuco, Brasil E-mail: junniorbiologia@hotmail.com

Luciana da Silva Bastos ORCID: https://orcid.org/0000-0002-7916-9911 Universidade Estadual do Maranhão, Brasil E-mail: lucianabastos79@gmail.com

Francisca Neide Costa

ORCID: http://orcid.org/0000-0002-8941-241X

Universidade Estadual do Maranhão, Brasil E-mail: francisca.cca.uema@gmail.com

Antonio Carlos Freitas Souza

ORCID: https://orcid.org/0000-0002-6921-9030 Instituto de Pesquisas Científicas e Tecnológicas do Estado do Amapá, Brasil E-mail: jr_bio2005@yahoo.com.br

\section{Resumo}

O consumo de carne bovina e bubalina está presente em vários lugares e culturas no mundo, principalmente por ser uma fonte importante de nutrientes de alto valor biológico, no entanto faz-se necessário que este alimento seja seguro, sem contaminações biológicas, físicas ou químicas. Desta forma o objetivo deste trabalho é avaliar as condições microbiológicas e físico-químicas das carcaças de bovinos e bubalinos dos dois abatedouros legalizados de Macapá, Amapá. Ao todo 50 amostras foram coletadas através de swabs e amostras de partes das carnes, juntamente a fiscalização da Agência de Defesa e Inspeção Agropecuária do Estado, posteriormente encaminhadas e analisadas nos laboratórios do Núcleo de Ciência e Tecnologia de Alimentos do Instituto de Pesquisas Científicas e Tecnológicas do Estado do Amapá (NUCTAL/IEPA) através dos métodos Adolfo Lutz (2008) A.O.A.C (2006) e MAPA (1999) seguindo os protocolos do referido núcleo que consiste na Instrução Normativa $\mathrm{N}^{\circ} 62$, do Ministério da Agricultura. Para as análises microbiológicas, as amostras apresentaram percentuais totais de 6\% para E. coli e 2\% para Samonella spp. No entanto, as maiores contaminações foram para Staphyloccocus aureus que chegaram a $50 \%$ do total das amostras. Com relação a composição centesimal, apesar de ambas as carnes apresentarem similaridades, notou-se 
grandes variações em proporção de gordura, que podem sofrer influência diante do seu conteúdo em aminoácidos essenciais e sua digestão.

Palavras-chave: Segurança de alimentos; Patógenos; Qualidade nutricional; Carcaças bovina e bubalinas.

\begin{abstract}
The consumption of beef and buffalo meat is present in several places and cultures in the world, mainly because it is an important source of nutrients of high biological value, however it is necessary that this food is safe, without biological, physical or chemical contamination. Thus, the objective of this work is to evaluate the microbiological and physicochemical conditions of bovine and buffalo carcasses from two legalized slaughterhouses in Macapá, Amapá. Altogether 50 samples were collected through swabs and samples of meat parts, together with the inspection of the State Agricultural Defense and Inspection Agency, later forwarded and analyzed in the laboratories of the Food Science and Technology Nucleus of the Scientific and Technological Research Institute. of the State of Amapá (NUCTAL/IEPA) through the methods Adolfo Lutz (2008) AOAC (2006) and MAPA (1999) following the protocols of the referred nucleus that consists of the Normative Instruction $\mathrm{N}^{\circ} 62$, of the Ministry of Agriculture. For the microbiological analysis, the samples presented total percentages of $6 \%$ for E. coli and $2 \%$ for Samonella spp. However, the greatest contaminations were for Staphylococcus aureus which reached 50\% of the total samples. Regarding the proximate composition, despite the fact that both meats have similarities, great variations in the proportion of fat were observed, which may be influenced by their content in essential amino acids and their digestion.
\end{abstract}

Keywords: Food safety; Pathogens; Nutritional quality; Bovine and buffalo carcasses.

\title{
Resumen
}

El consumo de carne de res y búfalo está presente en varios lugares y culturas del mundo, principalmente porque es una importante fuente de nutrientes de alto valor biológico, sin embargo es necesario que este alimento sea seguro, sin contaminación biológica, física o química. Así, el objetivo de este trabajo es evaluar las condiciones microbiológicas y fisicoquímicas de canales de bovinos y bufalinos provenientes de dos mataderos legalizados en Macapá, Amapá. En total se recolectaron 50 muestras a través de hisopados y muestras de partes cárnicas, junto con la inspección de la Agencia Estatal de Defensa e Inspección Agropecuaria, posteriormente remitidas y analizadas en los laboratorios del Núcleo de Ciencia y Tecnología de Alimentos del Instituto de Investigaciones Científicas y Tecnológicas de la Estado de Amapá (NUCTAL/IEPA) a través de los métodos Adolfo Lutz (2008) AOAC (2006) y MAPA (1999) siguiendo los protocolos del referido núcleo que consta de la Instrucción Normativa N62, del Ministerio de Agricultura. Para el análisis microbiológico, las muestras presentaron porcentajes totales de 6\% para E. coli y 2\% para Samonella spp. Sin embargo, las mayores contaminaciones fueron por Staphylococcus aureus que alcanzó el $50 \%$ del total de las muestras. En cuanto a la composición proximal, aunque ambas carnes presentan similitudes, se observaron grandes variaciones en la proporción de grasa, lo que puede estar influenciado por su contenido en aminoácidos esenciales y su digestión.

Palabras clave: Seguridad alimenticia; Patógenos; Calidad nutricional; Canales de vacuno y búfalo.

\section{Introdução}

Considerada como um dos principais alimentos da dieta humana, a carne tem seu papel na história da alimentação desde a era paleolítica, principalmente na Europa onde, a caça e o consumo da carne tiveram um aumento significativo, o que continuou desde a conquista do fogo até os dias de hoje (Flandrin \& Montanari, 2018)

O Instituto Brasileiro de Geografia e Estatística (IBGE, 2011) desde 2011 apresenta aumento de 1,6\% do efetivo nacional de bovinos em relação ao ano anterior, sendo concentrado no Centro-Oeste, Norte e Sudeste, enquanto o rebanho bubalino apresenta crescimento de 7,8\% nesse mesmo período, totalizando 1,3 milhões de cabeças, onde $38 \%$ são encontradas no estado do Pará e 18,4\% no estado do Amapá. Nos últimos dados, no Brasil, em 2019, após dois anos de queda, o rebanho bovino voltou a crescer com leve alta de $0,4 \%$ já o rebanho bubalino manteve-se em crescimento alcançando a quantidade de 1,4 milhões de cabeças (IBGE, 2019).

Com significativo crescimento do rebanho bubalino em comparação ao bovino, logo, observa-se que também o consumo de carnes de bubalinos tem aumentado entre a população amapaense, tendo em vista que o abate de bubalinos e bovinos acontecem diariamente nos abatedouros de Macapá, Amapá. No entanto Macêdo e Macêdo (2017) afirmam que a carne bubalina não é aceita por grande parte dos consumidores, mesmo apresentando características intrínsecas muito boas quando comparada a carnes de outras espécies. 
Mas além de seu papel histórico-cultural e características nutricionais o alimento cárneo por ser um alimento altamente perecível demanda muito cuidado sobre o processo higiênico-sanitário durante todas as fases de processamento, devido a alterações físicas, químicas e microbiológicas sofridas (Santos, Palma \& Santana 2017). Por isso garantir a higiene no manejo, local de produção e distribuição destes produtos é imprescindível para se obter um alimento seguro de substâncias e micro-organismos nocivos à saúde de todos (Santos et al., 2021).

A regionalidade das carnes pode apresentar resultados diferentes dos que já existem na literatura, tendo em vista os fatores que são, transporte adequado, atordoamento, abate e manejo que segundo Melo et al. (2016) repercutem de forma significativa na qualidade do produto. Por isso, o objetivo deste trabalho é avaliar as condições microbiológicas e físicoquímicas das carcaças de bovinos e bubalinos dos dois abatedouros legalizados de Macapá.

\section{Metodologia}

As amostras coletadas para estudo, foram adquiridas em matadouros localizados em 2 diferentes pontos da cidade de Macapá, no mês de agosto a novembro de 2021 com a colaboração Agência de Defesa e Inspeção Agropecuária do Estado do Amapá - DIAGRO. Para o estudo microbiológico, foram coletadas 50 amostras de material proveniente das carcaças (25 de bovinos e 25 de bubalinos) através de $S w a b$ acondicionados em tubos Falcon e para o estudo físico-químico, foram adquiridas amostras de carnes de forma aleatória, sem preferência entre os cortes, consequentemente obtendo-se as amostras de músculo e coração, armazenadas em sacos estéreis, ambos transportados em bolsas isotérmicas aos laboratórios do Núcleo de Ciência e Tecnologia de Alimentos do Instituto de Pesquisas Científicas e Tecnológicas do Estado do Amapá (NUCTAL/IEPA).

Na determinação microbiológica, os métodos analíticos foram baseados conforme Instrução Normativa $\mathrm{N}^{\circ} 60$ (Brasil, 2019) que estabelece métodos oficiais para análises microbiológicas de controle de produtos de origem animal e água. Os resultados encontrados, foram comparados com a legislação vigente para Escherichia coli; Staphylococcus aureus e Salmonella spp.

Para a identificação de E. coli, utilizou-se o pré-enriquecimento com $25 \mathrm{~g}$ da amostra em Caldo Lactosado por $24 \mathrm{~h}$ a $37^{\circ} \mathrm{C}$. Posterior ao período de incubação, foi realizada a identificação da bactéria através de uma alçada do homogeneizado em Caldo Soja Tripticaseína (TSB) e repicada pela técnica de esgotamento para placas contendo Ágar Eosina Azul de Metileno Levine (EMB - Levine) sendo incubadas a $36^{\circ} \mathrm{C} / 24 \mathrm{~h}-48 \mathrm{~h}$.

Após o período de incubação, foram selecionadas as colônias características (secas com brilho metálico) e repicadas em Ágar Nutriente para a conservação até o momento dos testes bioquímicos utilizados: Methyl Red e Voges-Proskauer (MRVP), Ágar Citrato de Simmons e Motilidade-Indol-Produção de Ácido Sulfídrico (SIM).

$\mathrm{Na}$ determinação de Staphylococcus aureus, uma alíquota de $25 \mathrm{~g}$ da amostra foi suspensa em $225 \mathrm{~mL}$ de salina peptonada e posteriormente transferida $0,1 \mathrm{~mL}$ para duplicatas de placas estéreis contendo $20 \mathrm{~mL}$ de Ágar Manitol Salgado. As placas foram incubadas a $35 \pm 2^{\circ} \mathrm{C} / 24-48 \mathrm{~h}$. As colônias típicas que apresentaram colônias circulares pequenas, convexas de coloração amarelado foram submetidas a prova de coagulase, por meio da transferência de $0,3 \mathrm{~mL}$ de plasma de coelho para um tubo de ensaio contendo Caldo Infusão Cérebro Coração (BHI) com incubação a $35 \pm 2{ }^{\circ} \mathrm{C}$, obtendo-se prova após 6 horas.

Para análise de Salmonella spp. procedeu-se o pré-enriquecimento homogeneizado de $25 \mathrm{~g}$ da amostra em $225 \mathrm{~mL}$ de Água Peptonada Tamponada (BPW) com incubação a $37 \pm 1^{\circ} \mathrm{C} / 18 \pm 2 \mathrm{~h}$. No enriquecimento seletivo, $0,1 \mathrm{~mL}$ foi transferido para $10 \mathrm{~mL}$ Rappaport-Vassilidis Soja (RVS) e $1 \mathrm{~mL}$ para $10 \mathrm{~mL}$ caldo Tetrationato Muller Kauffman novobiocina (MKTTn) e incubados a $41,5 \pm 1^{\circ} \mathrm{C} / 24 \pm 3 \mathrm{~h}$ e $37 \pm 1^{\circ} \mathrm{C} / 24 \pm 3 \mathrm{~h}$ respectivamente. Na fase de plaqueamento diferencial, para cada cultura de RVS

e do MKTTn foram estriadas uma alçada (estrias de esgotamento) em Ágar Xilose Lisina Desoxicolato (XLD) e incubadas a $35^{\circ} \mathrm{C}$ por $24 \mathrm{~h}$.

As análises físico-químicas foram baseadas nas metodologias do Instituto Adolfo Lutz (2008), A.O.A.C (2006) e 
MAPA (1999), obedecendo os protocolos padrões do Núcleo de Ciência e Tecnologia de Alimentos -- NUCTAL/IEPA, analisando os seguintes parâmetros: Proteína Total, Lipídios, Umidade, Cinzas e Carboidratos.

Para a determinação de proteínas, utilizou-se o método de Kjeldahl, através da digestão, destilação e titulação da amostra na determinação de nitrogênio total, através do aparelho de marca Tecnal, Modelo TE 036/1 (Zenebon, Pascuet \& Tiglea, 2008).

$\mathrm{Na}$ digestão, 1,5 $\mathrm{g}$ da mistura catalítica foi pesada em papel manteiga e $0,2 \mathrm{~g}$ da amostra colocada em tubo de Kjeldahl e levada ao bloco digestor, adicionado de $7 \mathrm{~mL}$ de ácido sulfúrico (H2S04), concentrado a temperatura gradativa de até $380^{\circ} \mathrm{C}$ por 3 horas. Após observação de tonalidade da solução na coloração azul-cristal, os tubos foram retirados do bloco digestor para esfriamento por cerca de 1 hora, em seguida adicionou-se lentamente água destilada permitindo o contato com a parede do tubo para que dobrasse a quantidade do volume inicial da solução.

No procedimento para destilação, foram adicionados $20 \mathrm{~mL}$ de ácido bórico $4 \%$, mais 2 gotas de indicador misto (vermelho de metila e verde de bromocresol) e levado para destilação junto de um tubo de Kjeldahl proveniente do bloco digestor. Adicionou-se $25 \mathrm{~mL}$ de hidróxido de sódio $(\mathrm{NaOH})$ a $50 \%$ em um copo acoplado ao aparelho para início de destilação até o ponto de coloração escura da solução em tubo.

Para a titulação, utilizou-se o Erlenmeyer proveniente do destilador que recebeu posteriormente a solução de ácido clorídrico (HCI) 0,1 molar, titulando até que o mesmo apresentasse mudanças em sua coloração. Na determinação, aplicou-se da seguinte maneira, a equação de nitrogênio total e proteína: Na determinação de nitrogênio total, aplicou-se a equação:

Nitrogênio total $(\%)=(0,14 \mathrm{x} \mathrm{Vg}$ xFator de correção $) /$ (Massa da amostra)

Eq. 1

Proteína $(\%)=\% \mathrm{Nx} 6$

Eq. 2

O método de Bligh e Dyer (1959) foi aplicado para a extração de lipídios à frio. Aproximadamente $10 \mathrm{~g}$ das amostras foram pesadas em Erlenmeyer de $250 \mathrm{~mL}$ e divididas em triplicatas, com adição de $50 \mathrm{~mL}$ de metanol, $25 \mathrm{~mL}$ de clorofórmio e $10 \mathrm{~mL}$ de água destilada, sendo misturada em agitador magnético por 20 minutos. Após o tempo de mistura, acrescentou-se mais $25 \mathrm{~mL}$ de clorofórmio e $25 \mathrm{~mL}$ de sulfato de sódio 1,5\%, agitando a amostra novamente por 2 minutos.

A solução posteriormente foi transferida para balão de decantação, onde ocorreu a formação de sistema bifásico onde removeu-se a fase orgânica contendo clorofórmio e lipídio para Erlenmeyer de $125 \mathrm{~mL}$ (A). Em um funil com papel de filtro, ocorreu a transferência da mistura do balão de decantação com certa quantidade de sulfato de sódio anidro, e filtrada em Erlenmeyer esterilizado e previamente pesado (B). A solução filtrada, seguiu para estufa da marca Quimis®, modelo Q-317 B222, para a evaporação total do solvente (C). A determinação total se deu através da seguinte equação:

Lipídios $(\%)=$ (Massa final-Massa inicial) $\times 100 /$ (Massa da amostra)

Eq. 3

Para umidade, o procedimento utilizado foi a partir da estufa da marca Quimis®, modelo Q-317 B222, onde foi utilizado o método de secagem em estufa com circulação de ar. Em um copo Becker pesado, esterilizado e resfriado em dessecador foi adicionado e pesado $3 \mathrm{~g}$ da amostra, em seguida levado para estufa à $105^{\circ} \mathrm{C}$ por 24 horas. Após o período, a amostra foi depositada em dessecador, pesada e levada novamente para estufa por 1hora até o alcance de peso constante obtendo o resultado através das seguintes equações:

$\begin{array}{ll}\text { Massa seca }(\%)=(\text { Massa final-Massa inicial) } \times 100 / \text { (Massa da amostra) } & \text { Eq. } 4 \\ \text { Umidade }(\%)=100 \text {-Massa seca. } & \text { Eq. } 5\end{array}$

Para determinação de resíduo mineral fixo foi utilizado o método de calcinação em mufla da marca Quimis®, modelo Q-318 D21, à temperatura de $550^{\circ} \mathrm{C}$. Pesou-se $3 \mathrm{~g}$ da amostra em cadinho previamente esterilizado, pesado e resfriado em dessecador, em seguida a amostra foi levada para mufla à $550^{\circ} \mathrm{C}$ durante 3 horas (Figura 3) e resfriados em dessecador até a obtenção de temperatura ambiente para pesagem. Para determinação de cinzas, utilizou-se a seguinte fórmula:

Cinzas $(\%)=(($ Massa Final - Massa Inicial $) \times 100) /($ Massa da Amostra $)$

Eq. 6 
A determinação de carboidratos foi obtida pela diferenciação de outras análises empregadas e calculada pela seguinte fórmula:

$$
\mathrm{E}=100-(\mathrm{A}+\mathrm{B}+\mathrm{C}+\mathrm{D})
$$

\section{Resultados e Discussão}

As análises de pesquisas microbiológicas para detecção das bactérias Salmonella spp., E. coli e S. aureus, feitas a partir das amostras de carcaças de bubalinos e bovinos dos dois abatedouros de Macapá, são apresentados na tabela 1.

Tabela 1. Resultados das análises de pesquisa microbiológicas de 50 amostras de carcaças de bovinos e bubalinos de abatedouros de Macapá, Amapá.

\begin{tabular}{cccc}
\hline Microrganismo & $\begin{array}{c}\text { Matadouro A } \\
25 \text { amostras }\end{array}$ & $\begin{array}{c}\text { Matadouro B } \\
25 \text { amostras }\end{array}$ & $\begin{array}{c}\text { Total } \\
50 \text { amostras }\end{array}$ \\
\hline E. coli & $4 \%$ & $8 \%$ & $6 \%$ \\
Salmonella spp & $4 \%$ & - & $2 \%$ \\
Staphylococcus aureus & $44 \%$ & $56 \%$ & $50 \%$ \\
\hline
\end{tabular}

Fonte: Autores (2021).

Os resultados apresentam no matadouro A, onde o abate de bubalinos foi predominante, 11 (44\%) foram positivas para S. aureus, 1 (4\%) positiva para Salmonella spp. e 1 (4\%) positiva para E. coli. No matadouro B, onde o abate predominante é de bovinos, 14 (56\%) foram positivas para S. aureus, 2 (8\%) positivas para E. coli e todas as amostras testaram negativo para Salmonella spp.

Diante destes resultados pode-se observar os perigos oferecidos a saúde da população, como observa o Ministério da Saúde (2021) onde diz que doenças causadas por alimentos, DTAs, são causadas por ingestão de alimentos e ou água contaminadas com bactérias e são um dos maiores causadores de morbidade e até mortalidade no mundo todo, sendo Salmonella spp., S. aureus e E. coli as principais causadoras.

A qualidade e segurança das carnes são fundamentais para a obtenção de um alimento seguro, no entanto, a integridade do alimento fica comprometida com a ausência de condições higiênico-sanitárias inadequadas influenciando direta ou indiretamente na saúde do consumidor (Silva, 2013).

No Amapá, produtos cárneos já foram avaliados do aspecto microbiológico, onde Souza et al. (2020) relataram a presença de coliformes termotolerantes e de Staphylococcus coagulase positiva em carnes moídas em açougues na zona sul da cidade de Macapá. Silva-Júnior et al. (2018) avaliando carne moída comercializadas nos supermercados da cidade de Macapá, também Staphylococcus coagulase positiva em todas as amostras analisadas.

Os dados do Sistema Nacional de Vigilância Epidemiológica das doenças Veiculadas por Alimentos VE-DTA (2019) demonstram que 5,3\% (2.350) dos surtos alimentares registrados no Brasil são provenientes de produtos cárneos, e dentre os agentes etiológicos, destacam-se a E. coli $(23,4 \%)$, a Salmonella spp. (11,3\%) e o S. aureus $(9,45 \%)$.

A carne por suas características nutricionais e grande atividade de água é um excelente meio de cultura para microorganismos, o que é agravado a partir do abate e processamento, quando os microrganismos externos como da microbiota da pele, trato digestivo, trato urinário entram em contato com o meio estéril que seriam a parte interna dos músculos (Fontoura, 2006). De acordo com Silva e Bueno (2018) no período que antecede o abate, diversas bactérias se encontram na pele do animal, juntamente com sujidades e material fecal o que acarreta a contaminação do local e na maior disseminação durante o abate. 
Segundo Moura et al. (2015) e Silva-Júnior et al. (2017) a contaminação por S. aureus em contagem alta aponta a decadência na higienização e sanitização dos equipamentos e manipuladores, além da necessidade de análise de pontos críticos de controle (APPCC) o que compromete a garantia de um alimento seguro para o consumidor.

O S. aureus é um micro-organismo oportunista e de grande potencial de causar doenças, tanto para os animais quanto para os humanos e são sempre associados aos surtos alimentares, o uso inapropriado e rotineiro de antimicrobianos tem levado ao aparecimento de cepas resistentes a essas drogas e a presença de S. aureus nas carcaças de bovídeos salienta a importância de cobrar sempre as condições higiênico-sanitárias dos manipuladores de forma a prevenir possíveis agravos na saúde da população (Gonçalves, 2015).

Concomitantemente, também foram avaliadas as características físico-químicas de cortes de bovinos e bubalinos, os resultados obtidos estão resumidos na Tabela 2 abaixo.

Tabela 2. Resultados das análises físico-químicas de amostras de carnes bovinas e bubalinas, sem distinção entre os cortes de bovinos e bubalinos.

\begin{tabular}{ccc}
\hline Nutrientes & Bovinos & Bubalinos \\
\hline Proténa (\%) & $17,52 \%$ & $15,39 \%$ \\
Lipídio (\%) & $1,00 \%$ & $5,60 \%$ \\
Umidade (\%) & $69,81 \%$ & $68,73 \%$ \\
Cinzas (\%) & $0,70 \%$ & $1,16 \%$ \\
Carboidrato (\%) & $10,92 \%$ & $9,12 \%$ \\
\hline
\end{tabular}

Fonte: Autores (2021).

Em relação a umidade, observou-se valores de $68,73 \%$ nas carnes bubalinas, enquanto a carne bovina apresentou média de 69,81\%. Os valores aproximaram-se ao obtido por estudos realizados por Andrighetto et al. (2005) em músculo Longissimus Dorsi de bubalinos. Para Velho et al. (2015), os resultados das análises em carne bovina in natura proveniente de estabelecimentos da cidade de Mossoró, Rio Grande do Norte, a variação ficou entre 60,16\% e 73,07\%, o que pode ter sido influenciado por clima quente seco e exposição de longo período. Os valores de umidade, refletem na conservação, maciez e suculência do alimento e o Zenebon et al. (2008) frisa que a carne possui aproximadamente $75 \%$ de seu peso em água, tendo variações de $65 \%$ a $80 \%$.

Para cinzas, as informações fornecem valores nutricionais quanto ao seu conteúdo mineral, em amostra bubalina observou-se a média de $1,16 \%$, e em bovinos o valor médio encontrado de $0,70 \%$. Em carnes bubalinas analisadas por Andrighetto et al. (2008) os valores se encontram com média a 1,6\% e 1,8\%. Para bovinos, Pitombo et al. (2008) relatou variação entre $1,04 \%$ a $1,6 \%$ em contrapartida, Livi (2015) chegou a resultados próximos a 2,38\% para carnes com cortes designados como primeira e segunda.

O teor lipídico obtido nas amostras de carnes bovinas ficou em média de 1,00\% e 5,60\% para bovinos e bubalinos respectivamente, no entanto seus percentuais podem sofrer variações de quantidade, quando relacionado a fatores como genótipo, idade e sexo, e em sua composição quando aliado a alimentação. Contudo, Oliveira (2005) ao comparar ambas as carnes, observou o diferencial lipídico em carnes bubalinas, que apresentaram menores teores de ácidos graxos saturados, dentre os mais indesejáveis, o ácido palmítico e esteárico e a presença de ácido graxos poliinsaturados, como o ômega 3 que os torna desejável em uma alimentação saudável.

Os resultados de carboidratos em carnes bovinas apresentaram percentuais de 10,92\% e 9,12\% para bovinos e bubalinos respectivamente, os valores podem sofrer variações quando relacionados ao corte. $\mathrm{O}$ valor proteico apresentou 
diferença de $2 \%$ na comparação das carnes estudadas, em bovinos a média de proteína chegou a 17,52\%, para bubalinos apresentou-se o valor de 15,39\%, sendo inferiores aos valores encontrados por Lira et al. (2005) em cortes bubalinos com $20,85 \%$.

\section{Considerações Finais}

Para o presente estudo, os resultados microbiológicos expressaram quantidades significativas para o micro-organismo S. aureus em ambos os matadouros e sucinta presença para Salmonella spp. e E. coli.

Os dados sugerem implantações de programas de autocontrole em boas práticas de manipulação de alimentos, procedimento operacional padrão e rotineira aplicação da metodologia de análises de perigos e pontos críticos de controle como medida de prevenção e controle higiênico sanitário durante o processo de abate e manipulação.

Sem deixar de lado os trabalhadores do ramo, visto que diante da pesquisa realizada, a bactéria encontrada em maior quantidade está presente na pele humana e fossas nasais além das peles e orifícios dos animais, tais medidas influenciarão diretamente na qualidade do produto final tornando o mais seguro para consumo.

Do ponto de vista nutricional ambas as carnes apresentaram teores aproximados na maior parte dos macronutrientes, enfatizando maiores diferenças em quantidades lipídicas o que demonstrou menor percentual em carnes bovinas, no entanto, diante de estudos presentes na literatura, o conteúdo de ácido graxo saturado se fez menos presente em carnes bubalinas, tendo em vista que o excesso de gordura saturada aumenta os níveis das taxas de colesterol no sangue, podendo assim ser considerado uma escolha de alimento saudável..

\section{Agradecimentos}

Agradecemos o apoio financeiro prestado pela FAPEAP/Decit/SCTIE/MS CNPq/SESA, através do edital Chamada pública N 003/2020 FAPEAP/Decit/SCTIE/MS/SESA-AP/CNPq - Programa Pesquisa para o SUS: Gestão compartilhada em saúde - PPSUS e ao Grupo de Pesquisa em Ciência e Tecnologia de Alimentos (NUCTAL/IEPA).

\section{Referências}

Almeida, J., Francisco, A., \& Silva, J. S. (2018). Qualidade da carne: breve abordagem dos aspectos intrínsecos, comerciais, tecnológicos, sensoriais e nutricionais. Dossiê Carnes. Tecno alimentar.

Andrighetto, C., Jorge, A. M., Roça, R., \& Rodrigues E. (2008). Características físico-químicas e sensoriais da carne de bubalinos Murrah abatidos em diferentes períodos de confinamento. Revista Brasileira de Zootecnia. 37 (12): 2179-2184.

Andrighetto, C., Athayde, N. B., Jorge, A. M., \& Francisco, C. L. (2005). Composição química e maciez do músculo longissimus dorsi de bubalinos jovens abatidos em diferentes tipos de confinamento. Congresso Brasileiro de Ciência e Tecnologia da Carne, 3, São Paulo: Instituto de Tecnologia de alimentos, p. $1-3$.

Brasil. (2019). Ministério da Saúde. Agência Nacional de Vigilância Sanitária. Padrões microbiológicos para alimentos. Instrução Normativa n 60 de 23 dezembro de 2019.

Brasil. (2010) Ministério da Saúde. Secretaria de Vigilância em Saúde. Departamento de Vigilância Epidemiológica. Manual Integrado de vigilância, prevenção e controle de doenças transmitidas por alimentos. Brasília: Editora do Ministério da Saúde.

Cora, F. M. (2016). Caracterização molecular de Escherichia Coli isoladas de bovinos, bubalinos e aves. Universidade Federal de Minas Gerais, UFMG. https://repositorio.ufmg.br/handle/1843/SMOC-A9SJX5.

Flandrin, J. L. \& Montanari, M. (2018). História da alimentação. (9a ed.), Estação liberdade.

Fontoura, C. L. (2016). Estudo microbiológico carcaças bovinas e influência da refrigeração sobre a microbiota contaminante. Universidade Estadual Paulista, Faculdade de Ciências agrárias e veterinárias. Jaboticabal, São Paulo, Brasil.

Gonçalves, A. P. (2015) Detecção de Staphylococcus aureus e perfil de resistência antimicrobiana em carcaças de bovinos abatidos em um frigorífico da região noroeste do Estado do Paraná. Universidade Paranaense. https://pesquisa.bvsalud.org/portal/resource/pt/vtt-202968

IBGE (2021). Instituto Brasileiro de Geografia e Estatística. https://cidades.ibge.gov.br/brasil/ap/pesquisa/46/0 
Lira, G. M., Mancini Filho, R. P., Torres, A. C. O., Vasconcelos, A. M. A., de Omena, C. M. B., \& de Almeida, M. C. S. (2015) Composição centesimal, valor calórico, teor de colesterol e perfil de ácidos graxos da carne de búfalo (Bubalis bubalis) da cidade de São Luiz do Quitunde-AL. Rev. Inst. Adolfo Lutz (Impr.), 64(1):31-38.

Livi, V. (2015). Avaliação microbiológica e físico-química de carne moída comercializada nos principais supermercados de Pato Branco - PR. 2015. 39 f. Trabalho de Conclusão de Curso (Graduação) - Universidade Tecnológica Federal do Paraná, Pato Branco. http://repositorio.utfpr.edu.br/jspui/handle/1/15317.

Macedo, L. P. C. A., \& Macedo, E. S. (2017). Carne de Búfalo: uma alternativa saudável. Animal Business Brasil. https://animalbusiness.com.br/medicinaveterinaria/tecnologia-de-alimentos/carne-de-bufalo-uma-alternativa-saudavel/.

Melo, A. F., Moreira, J. M., Ataídes, D. S., Guimarães, R. A. M., Loiola, J. L., \& Oliveira, R. Q. (2016). Fatores que influenciam na qualidade da carne bovina: Revisão. PUBVET. 10(10): 785-794.

Moura, E. S. R., Abrantes, M. R., Mendes, C. G., Oliveira, A. R. N., Sousa, E. S., \& Silva, J. B. A. (2015). Perfil higiênico-sanitário e perigos microbiológicos em abatedouros públicos. Rev. Bras. Med. Vet., 37(3): 203-208.

Oliveira, A. L. (2005) Búfalos: produção, qualidade de carcaça e de carne. Alguns aspectos quantitativos, qualitativos e nutricionais para promoção do melhoramento genético. Revista Brasileira de Reprodução Animal, 29(2): 122-134.

Pitombo, R. S., Souza, D. D. N., Ramalho, R. O. S., Figueiredo, A. B. A., Rodrigues, V. C., Freitas, D. D. G. C., \& Ferreira, J. C. S. (2013). Qualidade da carne de bovinos superprecoces terminados em confinamento. Arquivo Brasileiro de Medicina Veterinária e Zootecnia. 65(4): 1203-1207.

Santos, D. A., Amaral, G. V., Sartori, F. \& Simas, J. V. (2021) A importância das condições higiênico-sanitárias em abatedouros: Uma revisão de literatura. Research, Society and Development, 10(1):e22610111455.

Santos, R. L., Palma, J. M., \& Santana, A. P. (2017). Avaliação da qualidade higienicossanitária de carcaças de bovinos oriundos de abatedouros frigoríficos do Distrito Federal e entorno. Revista Higiene Alimentar. 31(272-273): 80-83.

Silva, C. C. S., \& Bueno, C. P. (2018). Pontos de contaminação de carcaças bovinas dentro do fluxograma de abate. Nutritime Revista Eletrônica.15(2): 81478152 .

Silva-Júnior, A. C. S., Ferreira, L. R., \& Frazão, A. S. (2017) Avaliação da condição higiênico-sanitária na comercialização de pescado da feira do Produtor Rural do Buritizal, Macapá-Amapá. LifeStyle Journal, 4(1): 71-81.

Silva-Júnior, A. C. S., Nascimento, J. F., Tostes, E. S. L., \& Silva, A. S. S. (2018). Análises microbiológicas de carne bovina moída comercializada em supermercados em Macapá, Amapá. PUBVET, 12(10): 1-7.

Souza, A. C. F., Viana, D. C., Souza, J. F., \& Costa, A. L. P. (2020). Análises físico-químicas e microbiológicas da carne moída comercializada em açougues de três bairros da Zona Sul de Macapá - Amapá. Research, Society and Development, 9(3):e182932708.

Velho, A. L. M. C. S., Abrantes, M. R., Medeiros, J. M. S., Aguiar, K. C. S., Souza, E. S., Soares, K. M. P., \& Silva, J. B. A. (2015). Avaliação qualitativa da carne bovina in natura comercializado em Mossoró-RN. Acta Veterinaria Brasilica, 9(3):212-217.

Zenebon, O., Pascuet, N. S., \& Tiglea, P. (2008). Métodos físico-químicos para análise de alimentos. (4a ed.), Instituto Adolfo Lutz. 